\title{
Identification of Potential Small Molecule Binding Pockets in p38 $\alpha$ MAP Kinase
}

\author{
Patricia Gomez-Gutierrez, ${ }^{\dagger, \$}$ Jaime Rubio-Martinez, $^{\S}$ and Juan J. Perez ${ }^{*} \stackrel{\ddagger}{ }$ \\ ${ }^{\dagger}$ Allinky Biopharma, Madrid Scientific Park, Faraday, 7, 28049 Madrid, Spain \\ ${ }^{\ddagger}$ Department of Chemical Engineering, Universitat Politecnica de Catalunya, ETSEIB. Av. Diagonal, 647, 08028 Barcelona, Spain \\ ${ }^{\S}$ Department of Physical Chemistry, Faculty of Chemistry, Universitat de Barcelona and Institut de Recerca en Quimica Teorica i \\ Computacional (IQTCUB), Marti i Franques 1-3, 08028 Barcelona, Spain
}

ABSTRACT: Given the essential role played by protein kinases in regulating cellular pathways, their dysregulation can result in the onset and/or progression of various human diseases. Structural analysis of diverse protein kinases suggests that these proteins exhibit a remarkable plasticity that allows them to adopt distinct conformations in response to interactions with other proteins, providing an opportunity for designing allosteric modulators. The present work reports the results of an in silico screening study aimed at identifying novel prospective allosteric binding sites in the paradigmatic p38 $\alpha$ MAP kinase. The process was carried out using a protein ensemble generated from a $6 \mu \mathrm{s}$ accelerated molecular dynamics simulation. The results of this calculation were first used to study the flexibility of the protein using Principal Component Analysis, followed by a Cluster Analysis aimed at producing an ensemble of conformations representative of the sampling process.

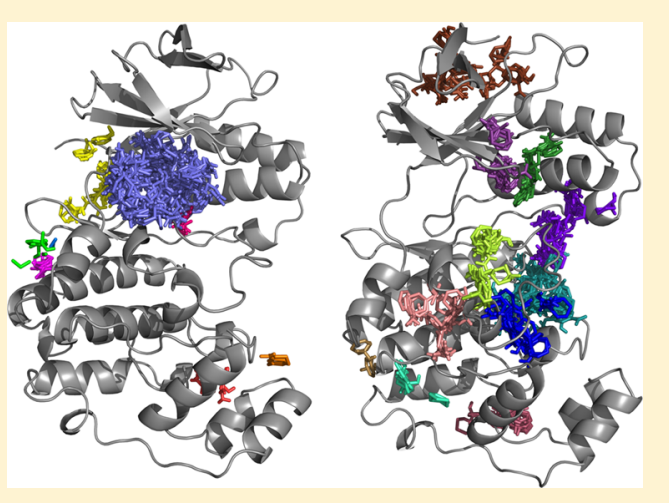
Representative structures of the diverse clusters were subsequently screened for hot spots using FTMAP. The procedure permitted the identification of diverse allosteric sites of p38 $\alpha$ already described in the literature including the DFG pocket, the lipid binding pocket, the DEF site, the docking groove, the $\mathrm{CD}$ and ED sites, and the backside site as well as a novel site recently reported: the A-loop regulatory site. Furthermore, the study also permitted the identification of ten novel prospective allosteric sites named NP1 to NP10, involving in most of the cases protein structural elements that control kinase activation including the activation loop, the catalytic loop, the $\alpha \mathrm{C}$ helix, the L16 loop, and the glycine-rich loop.

\section{INTRODUCTION}

Allosteric regulation is part of the intrinsic functioning of cells as well as a deregulation mechanism of many cellular processes. Allosterism is inherently connected to the plasticity of proteins $^{1,2}$ so that abnormalities affecting protein flexibility due to either environmental changes or internal perturbations such as mutations may impair their biological activity., Allosteric modulators are compounds that bind at a site different, often distant, from the active site to which the endogenous ligand binds. This regulatory mechanism paves the way for designing a new class of therapeutic agents. ${ }^{5}$

Protein phosphorylation is a paradigmatic example of allosteric modulation. It involves the transfer of the $\gamma$-phosphate group of ATP onto specific amino acids that exhibit a free hydroxyl group in substrate proteins and peptides. The transfer of the phosphate is associated with a conformational change in the structure of the substrate, causing it to become activated or deactivated. ${ }^{6}$ Protein phosphorylation is catalyzed by protein kinases, one of the largest families of enzymes with more than 500 members encoded in the human genome. ${ }^{7}$ As fundamental mediators of signaling pathways, dysregulation of protein kinases can result in dramatic changes in the control of many cellular processes resulting on the onset and/or progression of various human diseases including inflammatory, cardiovascular, metabolic, neurodegenerative diseases, and cancer. ${ }^{8}$

Due to their central role in cell signaling, protein kinases are major targets for therapeutic intervention. At present there are about 30 protein kinase inhibitors approved and more than a hundred in clinical trials, in addition to those in a preclinical state. ${ }^{9}$ The majority of approved inhibitors target the highly conserved catalytic ATP binding pocket. However, due to the high level of similarity of the site across family members, it is very challenging to achieve highly selective and potent compounds, required for the treatment of nonlife-threatening diseases like many immunological dysfunctions. In order to get more selective and potent inhibitors, interest has moved to discover allosteric inhibitors aimed at exploiting structural features and regulatory mechanisms that are unique to a particular kinase. ${ }^{10-15}$

The discovery of allosteric modulators has been traditionally associated with large high-throughput screening programs with limited success. Alternatively, computational techniques have demonstrated to be useful in identifying novel allosteric sites through the study of protein plasticity. ${ }^{16}$ Protein flexibility can

Received: July 26, 2017

Published: September 5, 2017 
be explained in terms of the rugged nature of the energy landscape that exhibits multiple low energy minima connected by low energetic barriers. Consequently, the inherent plasticity of proteins is the result of a dynamic exchange between different conformational substates that take place on a range of time scales from nanoseconds to seconds. ${ }^{17,18}$ Accordingly, the structure of a protein should not be viewed as rigid but as an ensemble of diverse conformations. ${ }^{19}$ More specifically, allostery can be understood as a redistribution of the conformational substates of the protein upon the perturbation exercised by the effector bound. ${ }^{20}$ Conformational sampling methods allow access to conformations which are not the most thermodynamically stable, but which are accessible at room temperature and are significant within the ensemble. ${ }^{21}$ Analysis of the diverse substates of a protein permits identification of transient pockets that can be exploited to alter its enzymatic activity via allosteric modulation.

The ultimate goal of the present study is the identification of prospective allosteric sites of the p38 $\alpha$ MAP kinase. For this purpose we carried out a detailed analysis of the flexibility of the enzyme using accelerated molecular dynamics (aMD) calculations. These studies were subsequently used to identify transient sites on the enzyme by means of an ensemble docking approach using a set of diverse small molecules as probes. As will be discussed later, the results of the present study suggest that the procedure is robust enough to identify diverse allosteric sites previously described in the literature and, furthermore, is capable of identifying ten novel prospective allosteric sites.

\section{METHODS}

Molecular dynamics (MD) simulations were carried out on the human p38 $\alpha$ MAP kinase. However, due to the lack of structures in the apo form with the A-loop completely solved, we selected the crystallographic structure of mouse p38 $\alpha$ (PDB ID 1 P38 $)^{22}$ due to its high resolution between residues 4 and $354(2.1 \AA)$. This structure corresponds to the unphosphorylated state and was solved without any ligand bound. In a subsequent step, residues 48 and 263 were mutated in silico to Leu and Thr, respectively, to get the sequence of the human p38 $\alpha$ by means of the MOE program. ${ }^{23}$ Finally, hydrogens were added to all protein residues to their corresponding protonation states at $\mathrm{pH} 7$, using the Protonate3D function of the MOE program. ${ }^{23}$ The mutation in silico of residues 48 and 263 does not produce any effect on the structure of the protein. To give support to this hypothesis we previously compared the structures 1P38 and 1A9U (human unphosphorylated p38 $\alpha$ crystallized bound to SB203580). The overlay of the two structures is good with an overall root-mean-square deviation (rmsd) of $0.253 \AA$. Moreover, the backbones of the two residues that are different in mouse and human p $38 \alpha$ sequence exhibit their side chains in the same orientation. Actually, the most important differences between the two crystal structures are found in the A- and P-loops that are located far from the position of the two residues.

The protein was soaked in a parallelepiped box of equilibrated TIP3P water molecules. After removal of those water molecules overlapping with the protein a total of 22,487 molecules remained. In addition, nine $\mathrm{Na}^{+}$ions were added to the solution to keep the neutrality of the system. Calculations were carried out with the ff99SB force field ${ }^{24}$ at $300 \mathrm{~K}$ using the NVT collective and periodic boundary conditions. A cutoff of $10 \AA$ was employed for the noncovalent interactions, and electrostatic interactions were treated using the PME method.
Before starting the molecular dynamics calculation the structure was energy minimized through 3000 steps using the steepest descent method followed by 1000 steps of the conjugate gradient method, in order to eliminate possible steric clashes. During the first 1000 minimization steps, harmonic positional restrictions of $1 \mathrm{kcal} / \mathrm{mol} \cdot \AA^{-2}$ were applied to all the atoms in the protein backbone. After minimization, the system was heated to $300 \mathrm{~K}$ using a molecular dynamics calculation within the NVT collective at a rate of $30 \mathrm{~K}$ per $10 \mathrm{ps}$. Subsequently, the system was equilibrated for $1 \mathrm{~ns}$ within the NPT collective, followed by $1 \mathrm{~ns}$ within the NVT collective, keeping the temperature at $300 \mathrm{~K}$ by means of the Langevin thermostat with a collision frequency of $2 \mathrm{ps}^{-1}$. The SHAKE algorithm was used in all the MD simulations to constrain all bonds involving hydrogen atoms to use an integration step of $2 \mathrm{fs}$.

Conformational sampling was performed by means of accelerated molecular dynamics for $6 \mu \mathrm{s}$ at $300 \mathrm{~K}$, following the protocol proposed by McCammon et al. ${ }^{25,26}$ and implemented in the Amber 14 program. ${ }^{27}$ Specifically, a biased potential was applied to both the total and dihedral torsional energies. The average potential energy (Ep) and the mean dihedral energy (Ed) as well as the parameters $\alpha \mathrm{P}$ and $\alpha \mathrm{D}$ were determined from a previous $50 \mathrm{~ns}$ classical molecular dynamics. After accelerated molecular dynamics calculations were completed, the effects due to the bias were removed by a ten order Maclaurin series reweighting of each configuration to recover the canonical ensemble. ${ }^{25}$ All the simulations were carried out using the pmemd program of the Amber12 software $^{27}$ on a graphics processing unit (GPU).

The results of the aMD calculation were subsequently used to characterize protein flexibility. For this purpose we first identified the invariant nucleus of the protein, defined by the set of residues that exhibit the smallest fluctuations along the MD trajectory. This was subsequently used to superimpose all $\mathrm{MD}$ snapshots and compute a covariance matrix to carry out Principal Component Analysis (PCA). ${ }^{28}$ This method filters thermal fluctuations and fast modes of local motions to reveal the slower, correlated modes of motions that are more likely associated with biological function. Furthermore, in order to identify the diverse conformations the protein attains, we carried out a hierarchical cluster analysis of the structures by means of the average link algorithm, ${ }^{29}$ using the backbone $\mathrm{C} \alpha$ root-mean-square deviation as a measure for the distance between two conformations. Finally, mapping of the prospective allosteric binding sites was carried out using the FTMap procedure. ${ }^{30}$ This method samples billions of positions of 16 different small organic molecules used as probes on a grid around the protein. Interaction energy is evaluated using first an empirical energy function, and then the best 2,000 poses are energy minimized using the CHARMM potential including solvation. A number of low energy poses are clustered, and the clusters are ranked on the basis of the average energy. The regions that bind several probe clusters are the predicted hot spots.

The druggabiliy of each of the prospective allosteric binding sites was assessed by means of the SiteMap program. ${ }^{31}$ The program characterizes the druggability of a binding site through the calculation of diverse properties that are evaluated by means of SiteScore and Dscore functions. More specifically, SiteScore measures the capacity of a pocket to bind ligands, whereas Dscore assesses the druggability, the druglike character of a pocket. Dscore allows the classification of a binding site as 
druggable (high values), difficult (intermediate values), and undruggable (low values).

\section{RESULTS AND DISCUSSION}

Conformational Analysis of $p 38 \alpha$. Analysis of the flexibility of p38 $\alpha$ was carried out using 600,000 structures extracted from the $6 \mu \mathrm{s}$ accelerated molecular dynamics calculation. Figure 1 shows the root-mean-square fluctuations

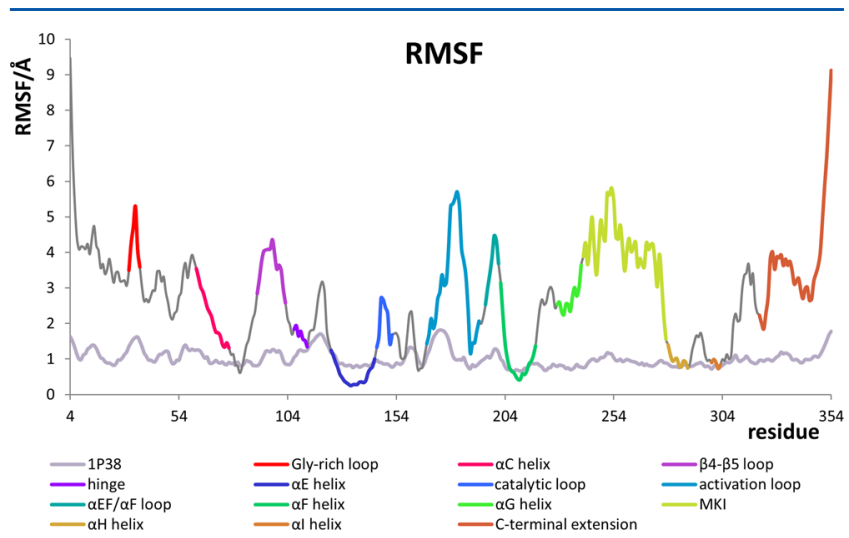

Figure 1. Root-mean-square fluctuations of $\mathrm{p} 38 \alpha \mathrm{C} \alpha$ atoms of all residues of $\mathrm{p} 38 \alpha$. In colors are those computed along the aMD trajectory, whereas in gray are those computed from the B-factors of the crystallographic structure according to the relationship rmsf $=[3$. $\left.B /\left(8 \pi^{2}\right)\right]^{1 / 2}$. Specific structural elements are highlighted in different colors.

(rmsf) of the different residues of the protein. For the sake of comparison is also shown the rmsf computed from the Bfactors of the crystallographic structure. As can be seen there is good qualitative agreement between the two lines. Present results on the rmsf of $\mathrm{p} 38 \alpha$ agree with those reported previously. ${ }^{32}$ Figure 2 shows pictorially the level of fluctuations of the $\mathrm{C} \alpha$ atoms of the diverse residues of $\mathrm{p} 38 \alpha$. As can be seen residues of the invariant nucleus correspond to the central residues of the $\alpha \mathrm{E}, \alpha \mathrm{F}$ helices depicted in blue and green. Analysis of Figures 1 and 2 suggests that the most flexible structural motifs of the protein include the glycine-rich loop, the C-terminal segment of the activation loop, the N-terminal end of the protein, the loop connecting helices $\alpha \mathrm{EF}$ and $\alpha \mathrm{F}$, and the $\beta 4-\beta 5$ loop, as well as the two structural elements specifically found in MAPKs: the MAP kinase insertion (MKI) motif and much of the C-terminal extension. Interestingly, many of these structural elements play an important role in p38 $\alpha$ function. Specifically, the activation loop plays an essential role in kinase activation, whereas the glycine-rich loop is believed to prevent the solvent from entering the catalytic site in addition to contributing to the positioning of the ATP phosphate groups. ${ }^{33}$ Furthermore, the MKI motif and the C-terminal extension are thought to be interfaces of the interaction with other proteins, ${ }^{34}$ so that the observed flexibility must play an important role in the formation of these proteinprotein interfaces. The MKI motif forms part of the interface or a coupling site known as DEF, whose accessibility would be coupled with the activation of the kinase, since the phosphorylation of this promotes site exposure. ${ }^{35,36}$ The allosteric inhibitor of JNK1 Compound 2 exerts its function binding to this site. ${ }^{10}$ Also, the MKI motif forms part of the lipid recognition pocket, an allosteric regulatory site in $\mathrm{p} 38 \alpha$ with Compound 10 as the only inhibitor known, ${ }^{10}$ and is

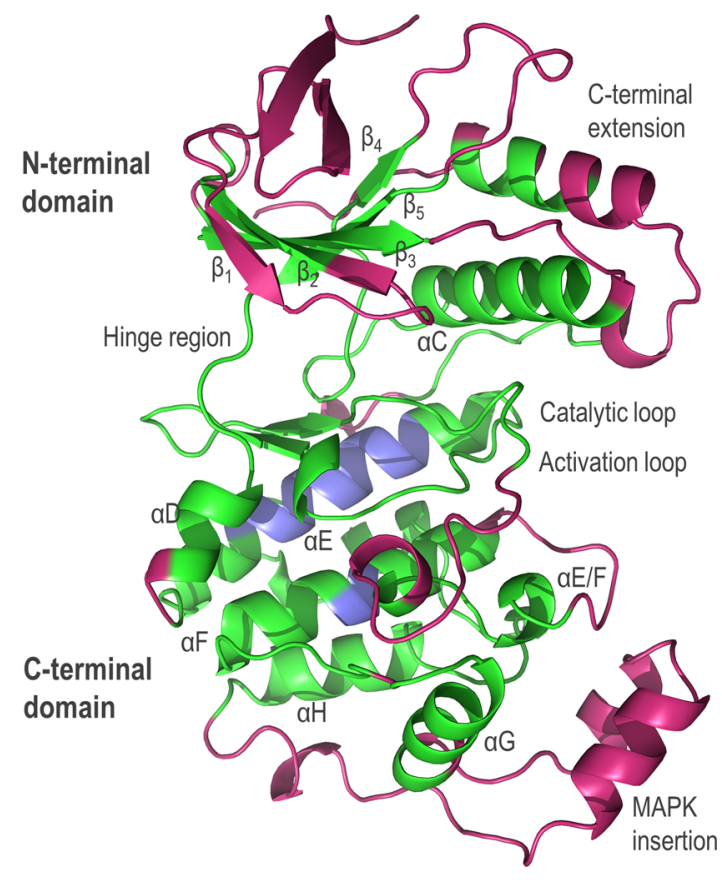

Figure 2. Flexibility of $\mathrm{p} 38 \alpha$. Residues with a rmsf of their $\mathrm{C} \alpha$ atoms lower than $0.5 \AA$ are shown in blue; residues with a rmsf between 0.5 and $3 \AA$ are shown in green, and residues with rmsf larger than $3 \AA$ are shown in purple.

believed to have a role in modulating the catalytic activity of the protein kinase in vivo. ${ }^{37,38}$ Finally, the C-terminal extension is a key element to keep MAPKs in an inactive conformation.

Other key domains involved in the regulation of p38 $\alpha$ activity exhibit intermediate flexibility including the catalytic loop, the $\mathrm{N}$-terminal segment of the activation loop, the $\alpha \mathrm{C}$ helix, and the hinge region. The catalytic loop contains residue Asp ${ }^{150}$ involved in the catalytic reaction of phosphorylation, while residues Lys ${ }^{152}$ and Asn ${ }^{155}$ in the same loop, as well as Asp $^{168}$ in the DFG motif of the N-terminal segment of the activation loop, participate in the positioning of the Mg-ATP. The position of the $\alpha \mathrm{C}$ helix is also key for the modulation of the catalytic activity, since the conserved $\mathrm{Glu}^{71}$ forces the position of $\mathrm{Lys}^{53}$ in the $\beta 3$-strand, which is also key in positioning the nucleotide at the active site. The hinge region that articulates the movement between the $\mathrm{N}$-terminal and the C-terminal domains controls the establishment of this important interaction between $\mathrm{Glu}^{71}$ and $\mathrm{Lys}^{53}$. A mechanism that regulates the formation of this interaction and prevents the intrinsic activity of $\mathrm{p} 38 \alpha$ is the interaction between the L16 loop at the C-terminal extension and the $\alpha \mathrm{C}$ helix.

Some structural features of the active conformation were not sampled in the accelerated molecular dynamics simulation. For instance, the active conformation of the activation loop found in the crystalline structures of phosphorylated p38 $\alpha$ was not sampled, suggesting that attaining this conformation without being phosphorylated must be considered as a rare event in the dynamics of the protein. ${ }^{39}$ This result agrees with experimental observation of the low level of basal activation of the nonphosphorylated kinase. The DFG-out conformation, found in the crystallographic structures of $\mathrm{p} 38 \alpha$ bound to type II inhibitors, is also not attained in the sampling process, although the movement of the motif is partially captured in one of the representative structures. This suggests that the binding mechanism of the ligands that occupy the allosteric DFG 
allosteric site may proceed through an induced fit mechanism as supported by steered molecular dynamics simulations. ${ }^{40}$ Alternatively, it could also be possible that significantly higher simulation times are necessary. Indeed, in order to sample the DFG-in to DFG-out transition previously reported $\mathrm{MD}$ simulations of $\mathrm{p} 38 \alpha$ were performed at $1000 \mathrm{~K}^{41}$

Principal Component Analysis. The Cartesian coordinates of the $\mathrm{C} \alpha$ corresponding to those residues with atomic fluctuations (rmsf) lower than $0.5 \AA$ (see Figure 1) define the invariant nucleus of the protein that was used to align the 600,000 structures collected during the sampling process. The alignment of structures aims to eliminate the rotational and translational movements in the subsequent analysis, focusing on the structural differences between the different states captured. The superposition of the structures permitted the recomputation of atom fluctuations to construct a covariance matrix that was subsequently diagonalized to obtain its principal components and eigenvalues. Visual inspection of the first five components, accounting for $63 \%$ of protein fluctuations, shows that only the first two, PC1 and PC2, involve whole protein motions (accounting for a $43 \%$ of protein fluctuations), whereas the three following involve motions at either the $\mathrm{N}$ - or the C-terminus. Specifically, the PC1 component corresponds to a bending motion involving the $\mathrm{C}$-terminal and $\mathrm{N}$-terminal lobes (see Figure 3a). The motion encompasses the entire $\mathrm{N}$ -
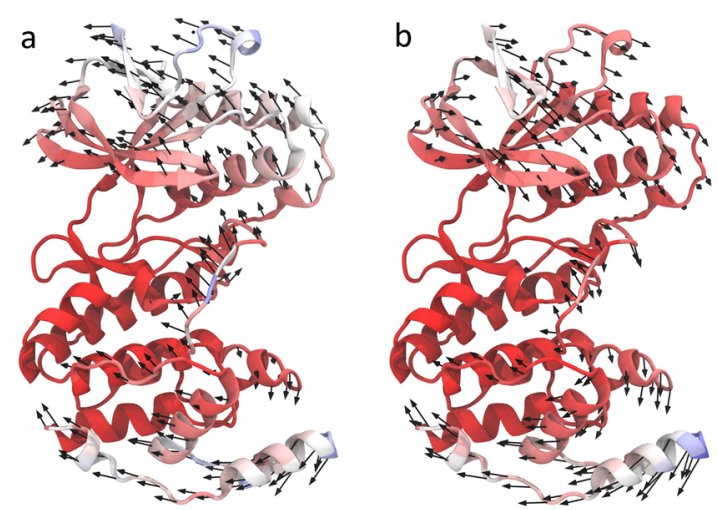

Figure 3. Representation of the two first principal components. The direction of the displacement of the $\mathrm{C} \alpha$ is shown by arrows, the length of the arrow being proportional to the magnitude of the vibration of the atom. For simplicity, the figure shows only arrows for vibrations that result in displacements greater than $1 \AA$. The protein skeleton is shown colored with a RWB (red to white to blue transition) gradient, in order of lowest to highest contribution of the skeleton of each amino acid to the major component. Calculations were carried out with the NMWiz module installed in the VMD program: ${ }^{66,67}$ a) representation of the PC1 and b) representation of the PC2.

terminal domain, while at the C-terminal domain involves the MKI motif, the $\alpha \mathrm{G}$ helix, the activation loop, and the $\alpha 1 \mathrm{~L} 14 /$ $\alpha \mathrm{H}$ loop. The PC2 component corresponds to a correlated rocking motion involving the same structural elements associated with the PC1 component, as can be seen in Figure 3b. Moreover, in the PC2 the MKI motif exhibits larger flexibility, while the $\mathrm{N}$-terminal domain exhibits a smaller fluctuation, especially at the $\alpha \mathrm{C}$ helix, the C-terminal extension, and the $\beta 4 / \beta 5$ loop. Similar findings in regard to the flexibility of $\mathrm{p} 38 \alpha$ were reported by Bakan et al. ${ }^{42}$ The following principal components involve one of the domains. Both components PC1 and PC2 are connected with the expected movements associated with $\mathrm{p} 38 \alpha$ functionality, including binding to the substrate and enzyme activation. PC3 and PC4 correspond to a rocking motion of the $\mathrm{N}$-terminus, and $\mathrm{PC} 5$ corresponds to a rocking motion of the $\mathrm{C}$-terminus similar to $\mathrm{PC} 1$, although the activation loop exhibits less amplitude.

Figure 4 shows pictorially the projection of the 600,000 snapshots onto the subspace defined by the two first principal

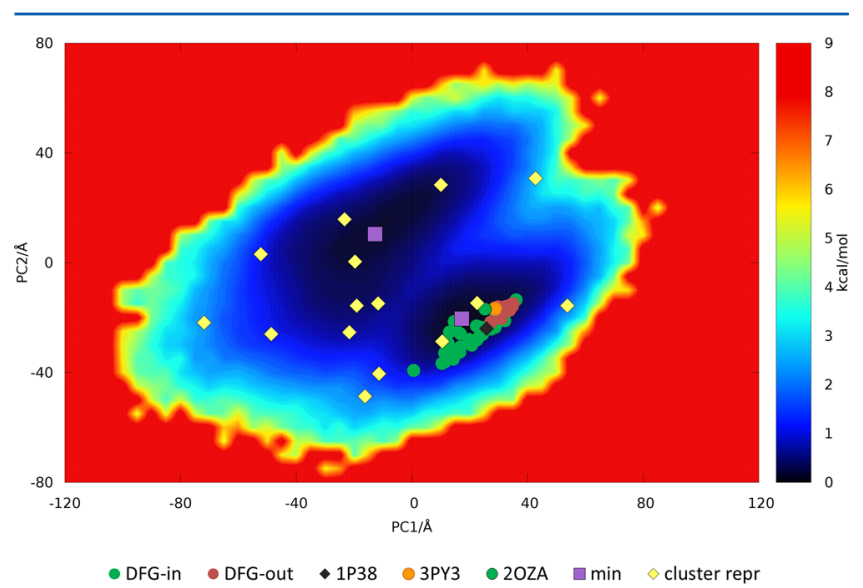

Figure 4. Free energy map calculated from the projection of the structures extracted from the aMD on the main components PC1 and PC2. It shows the projection of the coordinates of $\mathrm{p} 38 \alpha$ crystalline structures deposited in the PDB and completely resolved between amino acids 7 to 345 (PDB IDs: 1a9u, 1bl6, 1bl7, 1bmk, 1di9, 1m7q, louk, louy, love, 1p38, 1r3c, 1w7h, 1w84, 1wbw, 1yqj, 1zzl, 2i0h, 2lgc, 2yix, 2zb0, 3ds6, 3dt1, 3gc7, 3gc8, 3gfe, 3itz, 3py3, 3rin, 3s3i, 3u $8 w$, 4loo, 4lop, 1w82, 1w83, 1wbn, 1wbs, 1wbt, 1wbv, 3kq7, 3nnu, 3nnv, $3 \mathrm{nnw}$ ). In addition, the 2OZA structure has been included, whose phosphorylation loop was completed by homology modeling and molecular dynamics calculations. ${ }^{49}$ These structures have been divided into two groups with different colors: in green, structures whose activation loop is in a DFG-in conformation and in red, structures with the activation loop in a DFG-out conformation. The structure of the phosphorylated protein $3 \mathrm{PY} 3$ is represented by an orange circle. Finally, the coordinates of the energy minima of the conformational sampling and the representatives resulting from the analysis of clusters are indicated.

components (PC1 and PC2) after reweighting. Inspection of Figure 4 permits the identification of two basins in the low dimension representation of the free energy landscape. The lowest energy minimum (minimum 1) is located at coordinates $(17.5,-20.5)$ and represents 235 structures, whereas minimum 2 is found at coordinates $(-12.5,10.5)$ and represents 164 structures. In order to understand the conformational features of the structures representing these two minima, the corresponding average structures were generated and energy minimized. Comparison of the two structures shows a few structural differences. Specifically, structures located in minimum 2 are more open regarding the relative position of some structural elements such as the $\alpha \mathrm{C}$ helix, the C-terminal extension, and the MKI motif than in minimum 1 . Furthermore, the activation loop in minimum 2 is closer to the hinge region than in minimum 1 . In order to understand if the two minima represent active or inactive conformations and DFG-in or DFG-out structures, we projected onto the PC1/ PC2 map a set of 43 crystallographic structures of p38 $\alpha$ with residues 7 to 345 solved, covering differential structural features (see Figure 4). Interestingly, all the crystallographic structures are located in the minimum 1 basin. 
In order to get further insight into the features of the structures representing the two minima, we also analyzed the features of the regulatory spine (R-Spine) of the two structures. The R-spine is a structural motif consisting of five amino acids (notated as RS0-RS4), located at different protein structural elements whose relative position is considered to be essential for the catalytic activity of protein kinases. ${ }^{43,44}$ Thus, in the active conformation residue RS0 serves as an anchoring point to residues RS1-RS4 that are linked consecutively. Specifically, the side chain of RS0 interacts via a hydrogen bond with the $\mathrm{NH}$ group of the skeleton of the RS1 residue in the HRD motif. Moreover, residue RS1 interacts with residue RS2 of the conserved DFG motif in the activation loop, which also interacts at the active site with the $\mathrm{Mg}^{2+}$ ions that coordinate ATP, participating in its correct arrangement. Residue RS2 also interacts with residue RS3 of the $\alpha \mathrm{C}$ helix, whose position is vital for kinase activation, and residue RS3 interacts with the residue RS4 in the $\beta 4$ strand. Inactive conformations are those structures that have the R-Spine distorted. They can be classified into four groups (I-IV) depending on the position of the amino acid of the R-Spine displaced in respect to the active conformation. ${ }^{45}$ Thus, in the inactive conformation $\mathrm{I}$ the activation loop adopts a DFG-out conformation, which results in the disruption of the interactions of RS2 with residues RS1 and RS3, respectively. In the inactive conformation II the $\alpha \mathrm{C}$ helix moves to an $\alpha \mathrm{C}$-out conformation causing a disruption of the interaction between residues RS3 and RS2 and between residues RS3 and RS4, respectively. In the inactive conformation III there is a misalignment of residue RS1 with respect to the catalytically active conformation and the subsequent loss of the interaction between residues RS0 and RS1, resulting from the HRD-out conformation adopted by the kinase. Finally, the inactive conformation IV, or rotated lobes, occurs when the interaction between residues RS2 and RS3 is lost, as the result of the relative rotation of the two domains.

In $\mathrm{p} 38 \alpha$ the R-Spine is constituted by amino acids Asp $^{205}$ (RS0), $\mathrm{His}^{148}$ (RS1), Phe ${ }^{169}$ (RS2), Leu ${ }^{75}$ (RS3), and Leu ${ }^{86}$ (RS4). Figure 5 shows the position of these residues in the average structures of minima 1 and 2 , as well as in the crystalline structure 1P38. In the crystallographic structure the distance between the amino acids RS2 and RS3 is larger than in the active conformation due to the disposition of the activation loop and to the rotation of the $\mathrm{N}$ - and C-terminal domains. Accordingly, this structure can be considered as a type IV inactive conformation. Analysis of Figure 5 suggests that the average structure of minimum 1 exhibits the residues of $\mathrm{R}$ Spine in the same position to structure 1P38 and can consequently be considered as an inactive conformation of type IV. However, in the average structure of the minimum 2, the hydrogen bond interaction between the residues RSO and RS1 is lost due to the conformation adopted by the catalytic loop, suggesting that the structure can be classified as a type III inactive conformation.

Identification of Prospective Allosteric Binding Sites. As mentioned above, allostery is inherently connected to protein plasticity. Accordingly, in silico identification of allosteric binding sites requires the incorporation of protein flexibility in the screening process. This can be effectively done using an ensemble of conformations generated from a long MD calculation. ${ }^{46}$ For this purpose, we selected a few structures representing the 600,000 configurations extracted from the aMD simulation. The selection was carried out by means of the average linkage hierarchical clustering algorithm, ${ }^{29}$ using the

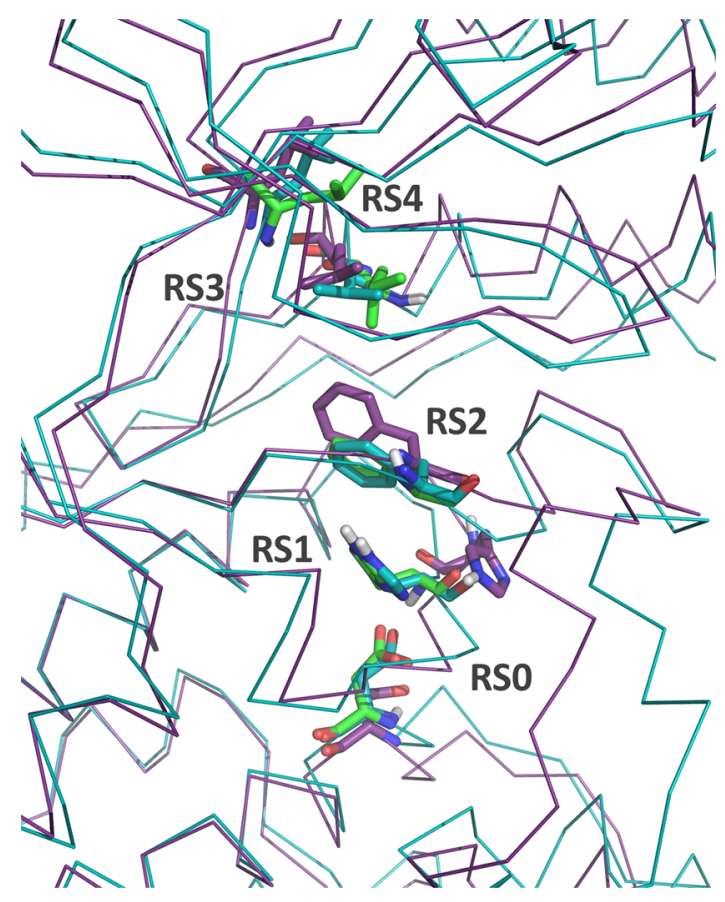

Figure 5. Position of the residues that constitute the R-Spine in minimum 1 (cyan), minimum 2 (purple), and in the crystal structure $1 \mathrm{P} 38$ (green).

rmsd of the $\mathrm{C} \alpha$ atoms as a measure of distance between two configurations. This procedure permitted grouping the configurations into 15 clusters that were represented by the structure with the closest rmsd to the average value, respectively. Figure 4 shows the location of the representative structures on the $\mathrm{PC} 1 / \mathrm{PC} 2$ map. The representative structures were subsequently used for screening prospective allosteric binding sites.

The surfaces of the 15 representative structures were mapped using the FTMap program. ${ }^{30}$ Figures $6 a$ and $6 \mathrm{~b}$ show the aggregated results of the 15 calculations where the diverse fragments are drawn for convenience on the crystalline structure used as starting structure for the MD calculations (PDB ID: 1P38). Shown in Figure 6a are those sites identified that have been previously described in the literature, whereas Figure $6 \mathrm{~b}$ shows prospective allosteric binding sites not previously described. Among the binding sites previously described in the literature, the ATP pocket is the most extensively sampled site including diverse fragments, being identified in all 15 representative structures. Inspection of the site shows that it includes, in addition to the ATP binding site, other adjacent pockets used by Type I inhibitors, such as the hydrophobic regions I and II. ${ }^{47}$ Moreover, despite the DFG-in/ DFG-out conformational transition not being observed during the simulation, in one of the representative structures the Nterminal segment of the activation loop appears slightly displaced in the direction of the C-terminal domain so that the FTMAP procedure is able to identify fragments that occupy the DFG pocket. The allosteric binding sites associated with the MKI motif, the lipid binding site, and the DEF site are also identified. ${ }^{47}$ There are fragments that dock in sites that overlap spatially with the structures of the allosteric ligands cocrystallized with the $\mathrm{p} 38 \alpha$ and JNK1 proteins. ${ }^{10}$ Fragments also bind to the docking groove, which is a site of protein-protein interaction that is presumed important in acquiring the active 

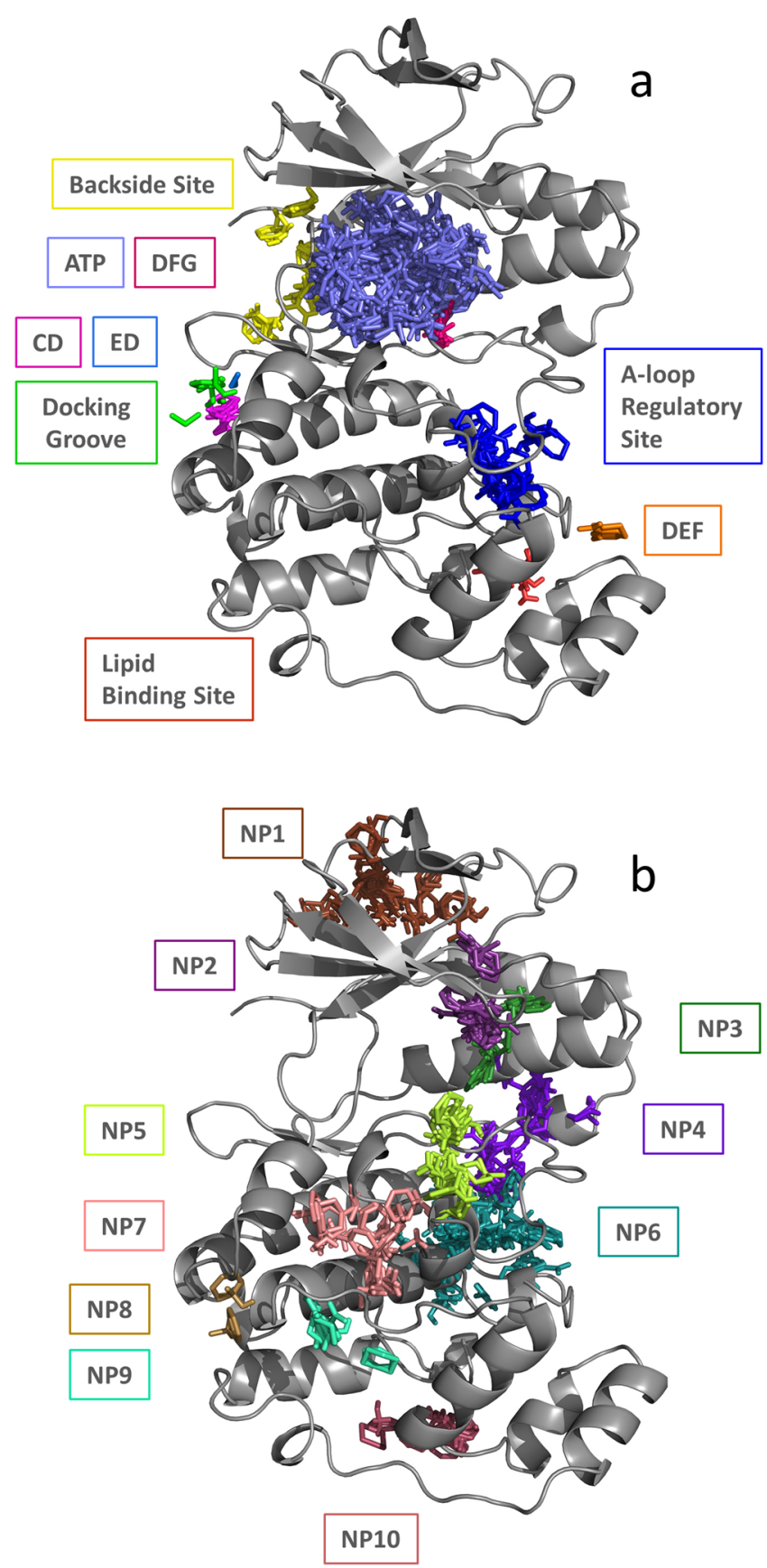

Figure 6. Aggregated probe map of the aMD trajectory 15 representatives bound to the 1P38 crystallographic structure. a) Sites already described in the literature: fragments binding to the ATP pocket and adjacent hydrophobic pockets I and II are shown in purple blue; fragments binding to the DFG pocket are shown in fuchsia; fragments binding to the union of lipids pocket are shown in red; fragments binding to the DEF site are shown in orange; fragments binding to the docking groove are shown in light green; fragments binding to the $\mathrm{CD}$ site are shown in magenta; fragments binding to the ED site are shown in light blue; fragments binding to the backside binding pocket are shown in yellow; and fragments binding to the Aloop regulatory site are shown in dark blue. b) Prospective sites not previously described in the literature.

conformation and acts as a regulator of signal duration and intensity, ${ }^{4-51}$ as well as its adjacent small ED and CD sites. Several inhibitors directed to these sites have been reported for ERK2 ${ }^{52,53}$ and JNK. ${ }^{54}$ The backside pocket that is suspected to be the site of the inhibitor of $\mathrm{p} 38 \alpha \mathrm{CMPD} 1^{55,56}$ appears in many of the structures. It is a wide pocket located behind the hinge region between the end of the $\mathrm{C}$-terminal extension and the turn that connects strands $\beta 7$ and $\beta 8$. Finally, the procedure also identifies an allosteric site recently reported by this laboratory, located between the ACE motif of the activation loop, the $\alpha \mathrm{EF}$ helix, the $\mathrm{N}$-terminal end of the $\alpha \mathrm{G}$ helix, and part of the $\mathrm{N}$-terminal segment of the activation. ${ }^{57}$

Additionally, ten prospective binding sites, not previously described for the p38 $\alpha$ protein or other MAP kinases named NP1 to NP10, are shown in Figure 6b. The NP1 site is located at the back of the beta sheets between the ends N-terminal and C-terminal, which is in an area with a high flexibility. This site appears more or less extensively in seven of the 15 representative structures. Also in the area of the beta sheets but next to the glycine-rich loop is the NP2 site, which is also observed in seven of the 15 selected structures. The glycine-rich loop or P-loop is an important structural motif in the development of catalysis since it is thought that it protects the solvent from the place where the catalytic reaction occurs and also contributes to the positioning of the phosphate groups of the ATP. ${ }^{33}$

The NP3 site is found in two of the representative structures in which the $\alpha \mathrm{C}$ helix adopts an inactive $\alpha \mathrm{C}$-out conformation. This conformation is characterized by the loss of the ionic pair between $\mathrm{Lys}^{53}$ ( $\beta 3$ strand) and $\mathrm{Glu}^{71}$ ( $\alpha \mathrm{C}$ helix), which is essential for kinase activity, that is interrupted due to $\alpha \mathrm{C}$ helix displacement. This site coincides with the pocket found in CDK2 using extrinsic fluorophore 8-anilino-1-naphthalenesulfonate (ANS) as probe. ${ }^{11,12}$ In addition, there are crystallographic structures of a few protein kinases, including EGFR, BRaf, MEK1/2, PDK1, and Akt1, bound to inhibitors in allosteric pockets arisen as a consequence of the $\alpha \mathrm{C}$ helix displacement that lie close to the NP3 site. Some of these inhibitors belong to the type IIB class, like for example the recently approved B-Raf inhibitors lapatinib and vemurafenib to treat breast cancer and advanced melanoma, respectively. ${ }^{13}$ These compounds can be considered as dual inhibitors that bind to the ATP and the NP3 sites. Other compounds that also bind close to the NP3 belong to the type III class of inhibitorsthey do not use the ATP pocket like for example trametinib, a recently approved inhibitor of MEK1 and MEK2. ${ }^{14}$ The NP4 site, also close to the $\alpha \mathrm{C}$ helix, extends toward the L16 loop, the catalytic loop, and the central segment of the activation loop. All of these are essential structural elements in the control of kinase activation or catalytic activity. The NP5 site is located in five of the representative structures. It is interesting that in one of these structures the activation loop is in a self-inhibited conformation in that it is folded toward the ATP site, partially occluding it as well as the catalytic loop. In this structure the activation loop adopts a conformation that bears some resemblance to that of the JNK3 structures with codes in the PDB 4H36, 4H39, and 4H3B. ${ }^{58}$

The NP6 site appears in ten of the 15 representative structures and is located behind the activation loop, encompassing the surface between the catalytic loop, the ACE motif of the activation loop, the $\alpha \mathrm{EF}$ helix, the $\alpha \mathrm{EF} / \alpha \mathrm{F}$ loop, and the N-terminal end of the $\alpha \mathrm{F}$ helix. The NP7 site appears in four of the representative structures and is located between the $\alpha \mathrm{D}$ helix, the $\alpha \mathrm{F}$ helix, the APE motif of the activation loop, the catalytic loop, and the $\alpha \mathrm{F} / \alpha \mathrm{G}$ loop. At the other side of this loop and close to the C-terminal end of the $\alpha$ F helix there is a prospective site, so-called NP9, that is found 
in two of the structures. Despite there being no inhibitor described to bind to this site, there is a crystallographic structure of $\mathrm{p} 38 \alpha$ that shows a fragment of the TAB1 protein bound to it that also occupies the docking groove. ${ }^{59}$ Binding of the peptide has effects on the conformation of the protein, so that the $\mathrm{N}$-terminal and $\mathrm{C}$-terminal lobes close up to each other as in the active conformation. Furthermore, the structure shows the activation loop in a conformation different to that found in other nonphosphorylated proteins, which is compatible with a cis autophosphorylation mechanism on the $\mathrm{Thr}^{180}$ residue. The activation loop of MK2 also binds to this site as shown in the crystallographic structure of the heterodimer $\mathrm{p} 38 \alpha / \mathrm{MK}^{60-62}$ and also is found as a prospective site by Bakan et al. ${ }^{63}$ and Coleman et al. ${ }^{64}$ On the other hand, in another protein family of MAPKs, JNK2, some of the amino acids of the $\alpha \mathrm{F} / \alpha \mathrm{G}$ loop and the latter of the $\alpha \mathrm{F}$ helix are involved in the autophosphorylation of this kinase. ${ }^{65}$ Near this site, between the $\mathrm{N}$-terminal end of the $\alpha \mathrm{E}$, the $\mathrm{C}$-terminal helix $\alpha \mathrm{F}$, and the $\mathrm{N}$-terminal helix $\alpha \mathrm{H}$ is located the NP8 site that appears in one of the structures obtained and which is also involved in the binding of the TAB1 protein fragment described above. Finally, the NP10 site is found in 3 of the representative structures, located at the $\mathrm{C}$-terminal ends of the $\alpha \mathrm{G}$ and $\mathrm{N}$-terminal helix of the $\alpha 2 \mathrm{~L} 14$ helix at the MAPK insertion and parallel to the loop connecting them both and extends to the C-terminal segment of the $\alpha \mathrm{H}$ helix.

The results of the druggability assessment using the SiteMap procedure are summarized in Table 1 . A value of SiteScore of

Table 1. Druggability Assessment for Each of the Binding Sites Identified in $\mathrm{p} 38 \alpha$ in the Present Work $^{a}$

\begin{tabular}{|c|c|c|}
\hline Sites & SiteScore & Dscore \\
\hline ATP & 1.156 & 1.07 \\
\hline Backside & 0.833 & 0.775 \\
\hline Docking Groove + ED + CD & 0.737 & 0.706 \\
\hline Lipid Binding & 1.08 & 1.104 \\
\hline A-loop Regulatory & 0.717 & 0.489 \\
\hline NP1 & 0.932 & 0.924 \\
\hline NP2 & 0.799 & 0.664 \\
\hline NP3 & 0.913 & 0.733 \\
\hline NP4 & 1.07 & 1.057 \\
\hline NP5 & 0.979 & 0.868 \\
\hline NP6 & 1.024 & 0.865 \\
\hline NP7 & 1.145 & 1.183 \\
\hline NP8 & 0.618 & 0.513 \\
\hline NP9 & 0.768 & 0.758 \\
\hline NP10 & 0.532 & 0.343 \\
\hline
\end{tabular}

${ }^{a}$ List of the SiteScore and Dscore values computed with SiteMap. The gray color indicates site with SiteScore $>0.80$, whereas green corresponds to values of Dscore $>0.98$; blue corresponds to the interval $0.98-0.83$.

0.80 is considered as an adequate threshold to discriminate between sites suitable for ligand binding with submicromolecular affinity from sites not able to bind ligands with high affinity. ${ }^{31}$ On the other hand, values of Dscore allow the classification of a binding site as druggable $(<0.98)$, difficult $(0.98-0.83)$, and undruggable $(>0.83)$. Inspection of Table 1 indicates that known binding sites such as the ATP pocket and the lipid binding site are well ranked using both parameters. In contrast, the docking groove, including the adjacent ED and the $\mathrm{CD}$ sites, is not well qualified as a binding site capable of binding druglike compounds with high affinity. Similar results are obtained for the recently reported A-loop regulatory site or the backside site. In these cases, it may be necessary to consider adjacent pockets in order to design new drugs targeting these sites. Regarding the novel sites identified in the present study, six of them have SiteScore values below the threshold: NP1, NP3, NP4, NP5, NP6, and NP7. Moreover, NP4 and NP7 are druggable according to the Dscore values, whereas NP1, NP5, and NP6 appear to be difficult sites and NP3 is not druggable. In this case, NP3 may be used to design dual ligands with high affinity using the proximal ATP and/or adjacent pockets used by type IIA and type IIB inhibitors.

\section{CONCLUSIONS}

The present work describes an analysis of the flexibility of the p38 $\alpha$ MAP kinase as deduced from the results of a $6 \mu \mathrm{s}$ accelerated MD calculation. PCA was used to analyze protein dynamics, and cluster analysis was used to select a set of a few configurations representing the ensemble. PCA suggests that the major components of the movement of the $\mathrm{p} 38 \alpha$ protein are related to relative movements of the $\mathrm{C}$-terminal and $\mathrm{N}$ terminal domains. While the first coordinate corresponds to the bending motion of the two domains, the second involves a rocking motion. Thus, both components describe the opening and closing movements of the interlobular space that are related to the activation-inactivation of protein kinases. Projection of the structures sampled along the MD trajectory onto the two first principal components provides the free energy surface that exhibits two minima. From the analysis of the R-Spine motif, it can be concluded that the lowest energy minimum (minimum 1) is represented by an inactive conformation type IV, while the minimum 2 is represented by a type III inactive conformation.

The representative structures from the cluster analysis were screened for hot spots using FTMap. The procedure permitted the identification of diverse allosteric sites in p38 $\alpha$ already described in the literature including the DFG pocket, the lipid binding pocket, the DEF site, the docking groove, the $\mathrm{CD}$ and ED sites, and the backside site as well as the A-loop regulatory site recently reported. In addition, ten novel prospective sites were identified on the surface of the $\mathrm{p} 38 \alpha$ protein (listed from NP1 to NP10), in most of the cases involving protein structural elements that control kinase activation including the activation loop, the catalytic loop, the $\alpha \mathrm{C}$ helix, the L16 loop, and the glycine-rich loop.

One of the novel sites identified (NP3) appears due to the adoption by the protein of the inactive $\alpha \mathrm{C}$-out conformation, which allows access to an allosteric site used by a type III inhibitor that binds to CDK2, that is adjacent to other allosteric pockets used by type IIB and type III kinase inhibitors. Inhibitors of the EGFR and B-Raf kinases, lapatinib and vemurafenib, and inhibitor of MEK1 and MEK2, trametinib, bind to these allosteric pockets and are currently approved by the FDA for the treatment of advanced breast cancer and melanoma. The present study suggests that this could be a good strategy for the inhibition of $\mathrm{p} 38 \alpha$, or even other proteins of the family of MAPKs, where type I inhibitors could be derived into the NP3 pocket. Another interesting finding is the identification of the prospective site (NP5), located in a selfinhibited conformation of $\mathrm{p} 38 \alpha$, in which the activation loop is folded toward the ATP site, which is similar to that present in a group of deposited JNK3 crystal structures in the PDB (PDB ID: $4 \mathrm{H} 36,4 \mathrm{H} 39$, and $4 \mathrm{H} 3 \mathrm{~B})$. The stabilization of this self- 
inhibited conformation by binding of a ligand at the NP5 site could also be exploited as a therapeutic strategy.

\section{AUTHOR INFORMATION}

\section{Corresponding Author}

*E-mail: juan.jesus.perez@upc.edu.

\section{ORCID}

Juan J. Perez: 0000-0002-0748-8147

\section{Notes}

The authors declare no competing financial interest.

The structures of the 15 representatives identified in the cluster analysis are available upon request from the authors.

\section{ACKNOWLEDGMENTS}

The authors are indebted to the scientific staff of Allinky Biopharma for helpful discussions.

\section{REFERENCES}

(1) Henzler-Wildman, K.; Kern, D. Dynamic Personalities of Proteins. Nature 2007, 450, 964-972.

(2) Bahar, I.; Lezon, T. R.; Yang, L. W.; Eyal, E. Global Dynamics of Proteins: Bridging Between Structure and Function. Annu. Rev. Biophys. 2010, 39, 23-42.

(3) Gorfe, A. A.; Grant, B. J.; McCammon, J. A. Mapping the Nucleotide and Isoform-Dependent Structural and Dynamical Features of Ras Proteins. Structure 2008, 16, 885-896.

(4) Nussinov, R.; Tsai, C. J. Allostery in Disease and in Drug Discovery. Cell 2013, 153, 293-305.

(5) Feixas, F.; Lindert, S.; Sinko, W.; McCammon, J. A. Exploring the Role of Receptor Flexibility in Structure-Based Drug Discovery. Biophys. Chem. 2014, 186, 31-45.

(6) Huse, M.; Kuriyan, J. The Conformational Plasticity of Protein Kinases. Cell 2002, 109, 275-282.

(7) Manning, G.; Whyte, D. B.; Martinez, R.; Hunter, T.; Sudarsanam, S. The Protein Kinase Complement of the Human Genome. Science 2002, 298, 1912-1934.

(8) Lahiry, P.; Torkamani, A.; Schork, N. J.; Hegele, R. A. Kinase Mutations in Human Disease: Interpreting Genotype-Phenotype Relationships. Nat. Rev. Genet. 2010, 11, 60-74.

(9) Fabbro, D. 25 Years of Small Molecular Weight Kinase Inhibitors: Potentials and Limitations. Mol. Pharmacol. 2015, 87, 766-775.

(10) Comess, K. M.; Sun, C. H.; Abad-Zapatero, C.; Goedken, E. R.; Gum, R. J.; Borhani, D. W.; Argiriadi, M.; Groebe, D. R.; Jia, Y.; Clampit, J. E.; Haasch, D. L.; Smith, H. T.; Wang, S. Y.; Song, D. Y.; Coen, M. L.; Cloutier, T. E.; Tang, H.; Cheng, X. H.; Quinn, C.; Liu, B.; Xin, Z. L.; Liu, G.; Fry, E. H.; Stoll, V.; Ng, T. I.; Banach, D.; Marcotte, D.; Burns, D. J.; Calderwood, D. J.; Hajduk, P. J. Discovery and Characterization of non-ATP Site Inhibitors of the Mitogen Activated Protein (MAP) Kinases. ACS Chem. Biol. 2011, 6, 234-244.

(11) Christodoulou, M. S.; Caporuscio, F.; Restelli, V.; Carlino, L.; Cannazza, G.; Costanzi, E.; Citti, C.; Lo Presti, L.; Pisani, P.; Battistutta, R.; Broggini, M.; Passarella, D.; Rastelli, G. Probing an Allosteric Pocket of CDK2 with Small Molecules. ChemMedChem 2017, 12, 33-41.

(12) Betzi, S.; Alam, R.; Martin, M.; Lubbers, D. J.; Han, H.; Jakkaraj, S. R.; Georg, G. I.; Schönbrunn, E. Discovery of a Potential Allosteric Ligand Binding Site in CDK2. ACS Chem. Biol. 2011, 6, 492-501.

(13) Wang, X.; Kim, J. Conformation-Specific Effects of Raf Kinase Inhibitors. J. Med. Chem. 2012, 55, 7332-7341.

(14) Palmieri, L.; Rastelli, G. $\alpha$ C Helix Displacement as a General Approach for Allosteric Modulation of Protein Kinases. Drug Discovery Today 2013, 18, 407-414.

(15) Dong, J.; Lu, W.; Pan, X.; Su, P.; Shi, Y.; Wang, J.; Zhang, J. Discovery of Novel Bcr-Abl Inhibitors Targeting Myristoyl Pocket and ATP Site. Bioorg. Med. Chem. 2014, 22, 6876-6884.
(16) Grant, B. J.; Lukman, S.; Hocker, H. J.; Sayyah, J.; Brown, J. H.; McCammon, J. A.; Gorfe, A. A. Novel Allosteric Sites on Ras for Lead Generation. PLoS One 2011, 6 (10), e25711.

(17) Frauenfelder, H.; Sligar, S. G.; Wolynes, P. G. The Energy Landscapes and Motion on Proteins. Science 1991, 254, 1598-1603.

(18) Wolynes, P. G. Symmetry and the Energy Landscapes of Biomolecules. Proc. Natl. Acad. Sci. U. S. A. 1996, 93, 14249-14255.

(19) Wei, G.; Xi, W.; Nussinov, R.; Ma, B. Protein Ensembles: How Does Nature Harness Thermodynamic Fluctuations for Life? The Diverse Functional Roles of Conformational Ensembles in the Cell. Chem. Rev. 2016, 116, 6516-6551.

(20) Swain, J. F.; Gierasch, L. M. The Changing Landscape of Protein Allostery. Curr. Opin. Struct. Biol. 2006, 16, 102-108.

(21) Bowman, G. R.; Geissler, P. L. Equilibrium Fluctuations of a Single Folded Protein Reveal a Multitude of Potential Cryptic Allosteric Sites. Proc. Natl. Acad. Sci. U. S. A. 2012, 109, 11681-11686.

(22) Wang, Z.; Harkins, P. C.; Ulevitch, R. J.; Han, J.; Cobb, M. H.; Goldsmith, E. J. The Structure of Mitogen-Activated Protein Kinase p38 at 2.1-Å Resolution. Proc. Natl. Acad. Sci. U. S. A. 1997, 94, 23272332.

(23) Molecular Operating Environment (MOE), 2013.08; Chemical Computing Group Inc.: 1010 Sherbooke St. West, Suite \#910, Montreal, QC, Canada, H3A 2R7, 2015.

(24) Hornak, V.; Abel, R.; Okur, A.; Strockbine, B.; Roitberg, A.; Simmerling, C. Comparison of Multiple Amber Force Fields and Development of Improved Protein Backbone Parameters. Proteins: Struct., Funct., Genet. 2006, 65, 712-725.

(25) Hamelberg, D.; Mongan, J.; McCammon, J. A. Accelerated Molecular Dynamics: a Promising and Efficient Simulation Method for Biomolecules. J. Chem. Phys. 2004, 120, 11919-11929.

(26) Pierce, L. C.; Salomon-Ferrer, R.; de Oliveira, C. A. F.; McCammon, J. A.; Walker, R. C. Routine Access to Millisecond Time Scale Events with Accelerated Molecular Dynamics. J. Chem. Theory Comput. 2012, 8, 2997-3002.

(27) Case, D.; Babin, V.; Berryman, J.; Betz, R.; Cai, Q.; Cerutti, D.; T.E. Cheatham, I.; Darden, T.; Duke, R.; Gohlke, H.; Goetz, A.; Gusarov, S.; Homeyer, N.; Janowski, P.; Kaus, J.; Kolossvary, I.; Kovalenko, A.; Lee, T.; LeGrand, S.; Luchko, T.; Luo, R.; Madej, B.; Merz, K.; Paesani, F.; Roe, D.; Roitberg, A.; Sagui, C.; Salomon-Ferrer, R.; Seabra, G.; Simmerling, C.; Smith, W.; Swails, J.; Walker, R.; Wang, J.; Wolf, R.; Wu, X.; Kollman, P. AMBER 14; University of California: San Francisco, 2014.

(28) Daidone, I.; Amadei, A. Essential Dynamics: Foundation and Applications. WIREs Comput. Mol. Sci. 2012, 2, 762-770.

(29) Rokach, L.; Maimon, O. Clustering methods. In Data Mining and Knowledge Discovery Handbook; Maimon, O., Rokach, L., Eds.; Springer: New York, NY, 2005; Chapter 15, pp 321-352, DOI: 10.1007/0-387-25465-X 15.

(30) Brenke, R.; Kozakovov, D.; Chuang, G. Y.; Beglov, D.; Hall, D.; Landon, M. R.; Mattos, C.; Vajda, S. Fragment-Based Identification of Druggable 'Hot Spots' of Proteins Using Fourier Domain Correlation Techniques. Bioinformatics 2009, 25, 621-627.

(31) Halgren, T. A. Identifying and Characterizing Binding Sites and Assessing Druggability. J. Chem. Inf. Model. 2009, 49, 377-389.

(32) Yang, Y.; Shen, Y.; Liu, H.; Yao, X. Molecular Dynamics Simulation and Free Energy Calculation Studies of the Binding Mechanism of Allosteric Inhibitors with p38 $\alpha$ MAP Kinase. J. Chem. Inf. Model. 2011, 51, 3235-3246.

(33) Huse, M.; Kuriyan, J. The conformational plasticity of protein kinases. Cell 2002, 109, 275-282.

(34) Pimienta, G.; Pascual, J. Canonical and Alternative MAPK Signaling. Cell Cycle 2007, 6, 2628-2632.

(35) Canagarajah, B. J.; Khokhlatchev, A.; Cobb, M. H.; Goldsmith, E. J. Activation Mechanism of the MAP Kinase ERK2 by Dual Phosphorylation. Cell 1997, 90, 859-369.

(36) Sheridan, D. L.; Kong, Y.; Parker, S. A.; Dalby, K. N.; Turk, B. E. Substrate Discrimination among Mitogen-Activated Protein Kinases through Distinct Docking Sequence Motifs. J. Biol. Chem. 2008, 283, 19511-19520. 
(37) Diskin, R.; Engelberg, D.; Livnah, O. A Novel Lipid Binding Site Formed by the MAP Kinase Insert in p38 alpha. J. Mol. Biol. 2008, $375,70-79$.

(38) Shaw, D.; Wang, S. M.; Villasenor, A. G.; Tsing, S.; Walter, D.; Browner, M. F.; Barnett, J.; Kuglstatter, A. The Crystal Structure of JNK2 Reveals Conformational Flexibility in the MAP Kinase Insert and Indicates Its Involvement in the Regulation of Catalytic Activity. J. Mol. Biol. 2008, 383, 885-893.

(39) Diskin, R.; Askari, N.; Capone, R.; Engelberg, D.; Livnah, O. Active Mutants of the Human p38alpha Mitogen-Activated Protein Kinase. J. Biol. Chem. 2004, 279, 47040-47049.

(40) Filomia, F.; De Rienzo, F.; Menziani, M. C. Insights into MAPK P38 $\alpha$ DFG Flip Mechanism by Accelerated Molecular Dynamics. Bioorg. Med. Chem. 2010, 18, 6805-6812.

(41) Frembgen-Kesner, T.; Elcock, A. H. Computational Sampling of a Cryptic Drug Binding Site in a Protein Receptor: Explicit Solvent Molecular Dynamics and Inhibitor Docking to p38 MAP Kinase. J. Mol. Biol. 2006, 359, 202-214.

(42) Bakan, A.; Bahar, I. The Intrinsic Dynamics of Enzymes Plays a Dominant Role in Determining the Structural Changes Induced upon Inhibitor Binding. Proc. Natl. Acad. Sci. U. S. A. 2009, 106, 1434914354.

(43) Kornev, A. P.; Haste, N. M.; Taylor, S. S.; Ten Eyck, L. F. Surface Comparison of Active and Inactive Protein Kinases Identifies a Conserved Activation Mechanism. Proc. Natl. Acad. Sci. U. S. A. 2006, 103, 17783-17788.

(44) Kornev, A. P.; Taylor, S. S.; Ten Eyck, L. F. A Helix Scaffold for the Assembly of Active Protein Kinases. Proc. Natl. Acad. Sci. U. S. A. 2008, 105, 14377-14382.

(45) Meharena, H. S.; Chang, P.; Keshwani, M. M.; Oruganty, K.; Nene, A. K.; Kannan, N.; Taylor, S. S.; Kornev, A. P. Deciphering the Structural Basis of Eukaryotic Protein Kinase Regulation. PLoS Biol. 2013, 11 (10), e1001680.

(46) Lin, J. H.; Perryman, A. L.; Schames, J. R.; McCammon, J. A. Computational Drug Design Accommodating Receptor Flexibility: The Relaxed Complex Scheme. J. Am. Chem. Soc. 2002, 124, 56325633.

(47) Fang, Z.; Grütter, C.; Rauh, D. Strategies for the Selective Regulation of Kinases with Allosteric Modulators: Exploiting Exclusive Structural Features. ACS Chem. Biol. 2013, 8, 58-70.

(48) Bardwell, A. J.; Frankson, E.; Bardwell, L. Selectivity of Docking Sites in MAPK Kinases. J. Biol. Chem. 2009, 284, 13165-13173.

(49) Chang, C. I.; Xu, B. E.; Akella, R.; Cobb, M. H.; Goldsmith, E. J. Crystal Structures of MAP Kinase p38 Complexed to the Docking Sites on its Nuclear Substrate MEF2A and Activator MKK3b. Mol. Cell 2002, 9, 1241-1249.

(50) Cuadrado, A.; Nebreda, A. R. Mechanisms and Functions of p38 MAPK Signalling. Biochem. J. 2010, 429, 403-417.

(51) Cuenda, A.; Rousseau, S. p38 MAP-Kinases Pathway Regulation, Function and Role in Human Diseases. Biochim. Biophys. Acta, Mol. Cell Res. 2007, 1773, 1358-1375.

(52) Hancock, C. N.; Macias, A.; Lee, E. K.; Yu, S. Y.; Mackerell, A. D., Jr.; Shapiro, P. Identification of Novel Extracellular SignalRegulated Kinase Docking Domain Inhibitors. J. Med. Chem. 2005, 48, 4586-4595.

(53) Chen, F.; Hancock, C. N.; Macias, A. T.; Joh, J.; Still, K.; Zhong, S.; MacKerell, A. D., Jr.; Shapiro, P. Characterization of ATPIndependent ERK Inhibitors Identified Through In Silico Analysis of the Active ERK2 Structure. Bioorg. Med. Chem. Lett. 2006, 16, 62816287.

(54) Stebbins, J. L.; De, S. K.; Machleidt, T.; Becattini, B.; Vazquez, J.; Kuntzen, C.; Chen, L. H.; Cellitti, J. F.; Riel-Mehan, M.; Emdadi, A.; Solinas, G.; Karin, M.; Pellecchia, M. Identification of a New JNK Inhibitor Targeting the JNK-JIP Interaction Site. Proc. Natl. Acad. Sci. U. S. A. 2008, 105, 16809-16813.

(55) Davidson, W.; Frego, L.; Peet, G. W.; Kroe, R. R.; Labadia, M. E.; Lukas, S. M.; Snow, R. J.; Jakes, S.; Grygon, C. A.; Pargellis, C.; Werneburg, B. G. Discovery and Characterization of a Substrate Selective p38alpha Inhibitor. Biochemistry 2004, 43, 11658-11671.
(56) Zhang, J.; Shen, B.; Lin, A. Novel Strategies for Inhibition of the p38 MAPK Pathway. Trends Pharmacol. Sci. 2007, 28, 286-295.

(57) Gomez-Gutierrez, P.; Campos, P. M.; Vega, M.; Perez, J. J. Identification of a Novel Inhibitory Allosteric Site in p38 $\alpha$. PLoS One 2016, 11 (11), e0167379.

(58) Laughlin, J. D.; Nwachukwu, J. C.; Figuera-Losada, M.; Cherry, L.; Nettles, K. W.; LoGrasso, P. V. Structural Mechanisms of Allostery and Autoinhibition in JNK Family Kinases. Structure 2012, 20, 21742184.

(59) De Nicola, G. F.; Martin, E. D.; Chaikuad, A.; Bassi, R.; Clark, J.; Martino, L.; Verma, S.; Sicard, P.; Tata, R.; Atkinson, R. A.; Knapp, S.; Conte, M. R.; Marber, M. S. Mechanism and Consequence of the Autoactivation of p38alpha Mitogen-Activated Protein Kinase Promoted by TAB1. Nat. Struct. Mol. Biol. 2013, 20, 1182-1190.

(60) Cumming, J. G.; Debreczeni, J. E.; Edfeldt, F.; Evertsson, E.; Harrison, M.; Holdgate, G. A.; James, M. J.; Lamont, S. G.; Oldham, K.; Sullivan, J. E.; Wells, S. L. Discovery and Characterization of MAPK-Activated Protein Kinase-2 Prevention of Activation Inhibitors. J. Med. Chem. 2015, 58, 278-293.

(61) ter Haar, E.; Prabakhar, P.; Liu, X.; Lepre, C. Crystal Structure of the p38 alpha-MAPKAP Kinase 2 Heterodimer. J. Biol. Chem. 2007, 282, 9733-9739.

(62) White, A.; Pargellis, C. A.; Studts, J. M.; Werneburg, B. G.; Farmer, B. T. Molecular Basis of MAPK-Activated Protein Kinase 2:p38 Assembly. Proc. Natl. Acad. Sci. U. S. A. 2007, 104, 6353-6358.

(63) Bakan, A.; Nevins, N.; Lakdawala, A. S.; Bahar, I. Druggability Assessment of Allosteric Proteins by Dynamics Simulations in the Presence of Probe Molecules. J. Chem. Theory Comput. 2012, 8, 24352447.

(64) Coleman, R. G.; Salzberg, A. C.; Cheng, A. C. Structure-based Identification of Small Molecule Binding Sites Using a Free Energy Model. J. Chem. Inf. Model. 2006, 46, 2631-2637.

(65) Cui, J.; Holgado-Madruga, M.; Su, W.; Tsuiki, H.; Wedegaertner, P.; Wong, A. J. Identification of a Specific Domain Responsible for JNK2alpha2 Autophosphorylation. J. Biol. Chem. 2005, 280, 9913-9320.

(66) Bakan, A.; Meireles, L. M.; Bahar, I. ProDy: Protein Dynamics Inferred from Theory and Experiments. Bioinformatics 2011, 27, $1575-1577$.

(67) Humphrey, W.; Dalke, A.; Schulten, K. VMD - Visual Molecular Dynamics. J. Mol. Graphics 1996, 14, 33-38. 